\title{
High-throughput identification of human SNPs affecting regulatory element activity
}

\author{
Joris van Arensbergen ${ }^{*}{ }^{1}$, Ludo Pagie ${ }^{1}$, Vincent D FitzPatrick ${ }^{2,3}$, Marcel de Haas ${ }^{1}$, Marijke P \\ Baltissen $^{4}$, Federico Comoglio ${ }^{1,5}$, Robin H van der Weide ${ }^{1}$, Hans Teunissen ${ }^{1}$, Urmo \\ Võsa ${ }^{6,7}$, Lude Franke ${ }^{6}$, Elzo de Wit ${ }^{1}$, Michiel Vermeulen ${ }^{4}$, Harmen J Bussemaker ${ }^{2,3}$, Bas \\ van Steensel ${ }^{*}, 1$
}

${ }^{1}$ Division of Gene Regulation, Oncode Institute, Netherlands Cancer Institute, Amsterdam, the Netherlands ${ }^{2}$ Department of Biological Sciences, Columbia University, New York, New York, USA ${ }^{3}$ Department of Systems Biology, Columbia University Medical Center, New York, New York, USA ${ }^{4}$ Department of Molecular Biology, Oncode Institute, Radboud Institute for Molecular Life Sciences, Radboud University Nijmegen, 6525 GA Nijmegen, The Netherlands ${ }^{5}$ Department of Haematology, University of Cambridge, Cambridge, UK ${ }^{6}$ Department of Genetics, University Medical Center Groningen, University of Groningen, Groningen, The Netherlands ${ }^{7}$ Estonian Genome Center, Institute of Genomics, University of Tartu, Tartu, Estonia

\section{Abstract}

Most of the millions of single-nucleotide polymorphisms (SNPs) in the human genome are noncoding, and many overlap with putative regulatory elements. Genome-wide association studies (GWAS) have linked many of these SNPs to human traits or to gene expression levels, but rarely with sufficient resolution to identify the causal SNPs. Functional screens based on reporter assays have previously been of insufficient throughput to test the vast space of SNPs for possible effects on regulatory element activity. Here, we leveraged the throughput and resolution of the SuRE reporter technology to survey the effect of 5.9 million SNPs, including 57\% of the known common SNPs, on enhancer and promoter activity. We identified more than 30,000 SNPs that alter the activity of putative regulatory elements, partially in a cell-type specific manner. Integration of this dataset with GWAS results may help pinpoint SNPs that underlie human traits.

\footnotetext{
Users may view, print, copy, and download text and data-mine the content in such documents, for the purposes of academic research, subject always to the full Conditions of use:http://www.nature.com/authors/editorial_policies/license.html\#terms

*Correspondence should be addressed to J.v.A. (joris.van.arensbergen@gmail.com) or B.v.S. (b.v.steensel@nki.nl). Author contributions

J.v.A. designed and performed experiments, analyzed data and wrote the manuscript. L.P., V.D.F. and H.J.B. developed algorithms and analyzed data. M.d.H. M.B., M.V., R.H.v.W., H.T., F.C., U.V., E.d.W. and L.F. generated and/or analyzed data. F.C. developed the web application. B.v.S. designed experiments, analyzed data and wrote the manuscript.

Competing interest statement

J.v.A. is founder of Gen-X B.V. (http://www.gen-x.bio/). E.d.W. is co-founder and shareholder of Cergentis B.V.. F.C. is a co-founder of enGene Statistics GmbH.
} 


\section{Introduction}

About 85 million SNPs have been identified in human genomes1. The vast majority of these are located in non-coding regions, and a typical human genome has about 500,000 variants with non-reference alleles overlapping regulatory elements such as enhancers and promoters1. It is becoming increasingly clear that such non-coding SNPs can have substantial impact on gene regulation 2 , thereby contributing to phenotypic diversity and a wide range of human disorders3-5.

GWAS and expression quantitative trait locus (eQTL) mapping can identify candidate SNPs that may drive a particular trait or disorder6,7 or the expression level of individual genes 3,8 , respectively. Unfortunately, even the largest GWAS and eQTL studies rarely achieve singleSNP resolution, largely due to linkage disequilibrium (LD). In practice, tens to hundreds of linked SNPs are correlated with a trait. Although new fine-mapping techniques9-11, integration with epigenomic data12, deep learning computational techniques 13 and GWAS of extremely large populations can help to achieve higher resolution, pinpointing of the causal SNPs remains a major challenge.

Having a list of all SNPs in the human genome that have the potential to alter gene regulation would mitigate this problem. Ideally, the regulatory impact of SNPs would be measured directly. Two high-throughput methods have been employed for this purpose. First, changes in chromatin features such as DNase sensitivity and various histone modifications have been mapped in lymphoblasts or primary blood cells derived from sets of human individuals with fully sequenced genomes14-20. Here, the chromatin marks serve as proxies to infer effects on regulatory elements, with the caveat that a change in regulatory activity may not always be detected as a change in chromatin state, or vice versa. Furthermore, many traits do not manifest in blood cells, and other cell types are more difficult to obtain for epigenome mapping.

An alternative functional readout is to insert DNA sequence elements carrying each allele into a reporter plasmid. Upon transfection of these plasmids into cells, the promoter or enhancer activity of these elements can be measured quantitatively. Different cell types may be used as models for corresponding tissues in vivo. Large-scale versions of this approach are referred to as Massively Parallel Reporter Assays (MPRAs), which have been applied to screen tens of thousands of SNPs21-25. Each of these studies has yielded tens to at most several hundreds of SNPs that significantly alter promoter or enhancer activity. As these MPRA studies have covered only a tiny fraction of the genome, it is likely that many more SNPs with regulatory impact are to be discovered.

Here, we report application of an MPRA strategy with a >100-fold increased scale compared to previous efforts. This enabled us to survey the regulatory effects of 5.9 million SNPs in two different cell types, providing a resource that helps to identify causal SNPs among candidates generated by GWAS and eQTL studies. The data are available for download, and can be queried through a web application (https://sure.nki.nl). 


\section{Results}

\section{A survey of 5.9 million SNPs using SuRE}

We applied our Survey of Regulatory Elements (SuRE) technology to systematically screen millions of human SNPs for potential effects on regulatory activity. SuRE is an MPRA with sufficient throughput to query entire human genomes at high resolution and high coverage26. Briefly, random genomic DNA (gDNA) fragments of a few hundred base pairs are cloned into a promoter-less reporter plasmid that, upon transfection into cultured cells, only produces a transcript if the inserted gDNA fragment carries a functional transcription start site (TSS) (Fig. 1a). The transcript is identified and quantified by means of a random barcode sequence that is unique for every insert, allowing for a multiplexed readout of millions of random DNA fragments. Importantly, because active promoters as well as enhancers generate transcripts, activity of both types of elements can be assayed quantitatively by SuRE26.

To survey a large cross-section of SNPs present in the human population, we chose four sequenced genomes of individuals from 4 different populations1 (Fig. 1b). From each genome we generated two independent SuRE libraries that each contained $~ 300$ million random gDNA fragments of 150-500 bp (Supplementary Fig. 1a; Supplementary Table 1). In these libraries a total of 2,390,729,347 unique gDNA fragments were sequenced from both ends, mapped to the reference genome and linked to their unique barcode. Among these fragments, 1,103,381,066 carried at least one SNP for which we identified both alleles in our libraries. These libraries enabled us to test promoter/enhancer activity of both alleles of $5,919,293$ SNPs, which include 4,569,323 (57\%) of the $\sim 8$ million known common SNPs (minor allele frequency $[\mathrm{MAF}]>5 \%$ ) world-wide1. Importantly, each SNP allele is covered by 122 different gDNA fragments on average (Supplementary Fig. 1b,c), which provides substantial statistical power.

We introduced these libraries by transient transfection into human K562 and HepG2 cell lines. K562 is an erythroleukemia cell line with strong similarities to erythroid progenitor cells23. HepG2 cells are derived from a hepatocellular carcinoma, and serve as an approximate representation of liver cells. After transfection of the SuRE libraries into each cell line we isolated mRNA and counted the transcribed barcodes by Illumina sequencing. Three independent biological replicates yielded a total of 2,377,150,709 expressed barcode reads from K562 cells and two biological replicates yielded 1,174,138,611 expressed barcode reads from HepG2 cells.

\section{Identification of thousands of SNPs with regulatory impact}

From these data we first constructed strand-specific tracks of SuRE enrichment profiles for each of the four genomes (Fig. 1b). This revealed thousands of peaks that generally colocalize with known enhancers and promoters (Supplementary Fig. 1d), as reported previously26. For a subset of peaks, the magnitude varied between the four genomes and showed a correlation with a particular allele of a coinciding SNP. For example, in K562 cells we detected a strong SuRE signal overlapping with SNP rs6739165 in the genomes that are 
homozygous for the $\mathrm{C}$ allele, but no signal in the genome that is homozygous for the $\mathrm{A}$ allele, and an intermediate signal in the genome that is heterozygous for this SNP (Fig. 1b).

In order to systematically annotate SNPs we combined for each transfected cell line the complete SuRE datasets from the four genomes. The sequencing data of the SuRE libraries then enabled us to group for each SNP the overlapping gDNA fragments by the two alleles (Fig. 1c,d). This allowed us to identify SNPs for which fragments carrying one allele produced significantly different SuRE signals compared to those carrying the other allele. Because all of these fragments differ in their start and end coordinate, the activity of each allele is tested in a multitude of local sequence contexts, providing not only statistical power but also biological robustness. For each SNP we calculated a $P$ value, and we used a random permutation strategy to estimate false discovery rates (FDR) (Supplementary Fig. 1e,f). We also required that the strongest allele showed an average SuRE signal of at least 4-fold over background. We refer to the resulting SNPs at FDR $<5 \%$ as reporter assay QTLs (raQTLs).

This analysis yielded a total of 19,237 raQTLs in K562 cells and 14,183 in HepG2 cells (Fig. 1e). The average allelic fold change of these SNPs was 4.0-fold (K562) and 7.8-fold (HepG2) (Supplementary Fig. 1g,h). In 72\% of cases the SuRE effect could be assigned to a single SNP; when SNPs were spaced less than $\sim 200$ bp apart, their effects could typically not be resolved (Supplementary Fig. 1i).

Most raQTLs were detected in either K562 or HepG2 cells, but not in both (Fig. 1e). The overlap may be underestimated due to the somewhat arbitrary FDR and expression cutoffs used to define these sets (Supplementary Fig. 1j). Nevertheless, many SNPs show clear cell type specific effects (Supplementary Fig. 1j-1). For example, rs4265625:G creates regulatory activity in HepG2 only (Fig. 1f). This is interesting, because rs4265625 lies in POU2AF1, a gene that has been linked to primary biliary cirrhosis - a liver disease - in a GWAS27. In about 1,300 instances, a K562-specific raQTL and a HepG2-specific raQTL are in strong LD $\left(\mathrm{R}^{2}>0.8\right)$. An interesting possibility is that the two raQTLs in such linked pairs may contribute to the regulation of a common target gene, but each in a cell type specific manner.

\section{raQTLs are enriched for known regulatory elements}

We systematically analyzed the overlap of the raQTLs with known regulatory elements in K562 cells28. Compared to all SNPs analyzed, raQTLs showed 5-15 fold enrichment for promoter and enhancer related chromatin types, and depletion for repressed or transcribed chromatin types (Fig. 2a). We also observed strong enrichment of raQTLs in DNase hypersensitive sites (DHS) (Fig. 2b,c). This is consistent with SuRE signals overlapping with enhancers or promoters, as shown previously 26.

Some of the raQTLs are heterozygous in the genome of K562 cells. For these SNPs we investigated whether the allelic imbalance observed by SuRE was reflected in a corresponding imbalance in the DHS signal. For example, the SuRE signal at rs12985827, a non-coding SNP in an intron of the $A P C 2$ gene, has a strong bias for the $\mathrm{C}$ allele (the alternative allele, ALT) over the T allele (the reference allele, REF) (Fig. 2d). Indeed, it shows a similar allelic imbalance for DHS (Fig. 2e). Among the 616 raQTLs that were heterozygous in K562 and showed sufficient DNase-seq coverage, we observed a strong 
skew for higher DNase sensitivity at the more active allele, compared to 616 heterozygous non-raQTL control SNPs that overlapped DHSs (Fig. 2f,g). We found a similar but less pronounced trend in H3K27ac28 and ATAC-seq29 data from K562 cells (Supplementary Fig. 2). We conclude that available epigenomic data is generally consistent with the SuRE results.

\section{Altered transcription factor binding sites at raQTLs}

The observed changes in enhancer or promoter activity are likely explained by SNPs changing transcription factor (TF) binding motifs. For example, the T allele of rs 12985827 disrupts an EGR1 binding motif and leads to reduced SuRE activity (Fig. 3a, Fig. 2d). To investigate this systematically, we made use of the computationally predicted changes in TF motifs in the SNP2TFBS database30. Among the set of raQTLs in K562 and HepG2 cells, $31 \%$ and $38 \%$ are predicted to alter the motif of at least one TF, respectively. This is a 1.6fold and 1.9-fold larger proportion than for all SNPs, respectively (Fig. 3b). Moreover, 67\% and $69 \%$ of the raQTLs showed concordance between the predicted effect on motif affinity and SuRE expression, i.e., the allele with the weakest motif resemblance had the lowest SuRE expression (Fig. 3c). We note that $100 \%$ concordance should not be expected in this analysis, for example because not all TF binding motifs are known, some may be misannotated, and some TFs can act as repressors.

We expected that motifs in raQTLs reflect the sets of TFs that are selectively active in the respective cell types. Indeed, raQTLs in K562 were enriched for motifs of TFs that are primarily active in the erythroid blood lineage, such as GATA and STAT factors, while raQTLs in HepG2 cells were enriched for motifs of TFs that are specific for liver cells, such as HNF factors (Fig. 3d). Disruptions of the TP53 motif also appeared to be relatively more consequential in HepG2, which is presumably related to the known inactivation of TP53 in K562 cells31 but not in HepG2 cells32. Together, these data point to a general concordance between the detected changes in SuRE activity and predictions based on sequence motif analysis.

\section{No evidence for strong negative selective pressure on raQTLs}

It has been observed that genes that do not have cis-eQTLs are more likely to be loss-offunction (LOF) intolerant genes, possibly reflecting selection against variants acting upon such genes8,33. We found that the fraction of SNPs that are raQTLs was significantly but only slightly lower in the proximity of LOF intolerant genes than in the proximity of LOF tolerant genes (Supplementary Fig. 3a,b). However, for a set of control SNPs that were matched to the raQTLs for their SuRE activities and coverage in the SuRE libraries we observed a similar pattern (Supplementary Fig. 3a,b). This suggests that the overall density of active regulatory elements, rather than elements affected by SNPs, is lower near LOF intolerant genes. Genome-wide, we observed slightly lower minor allele frequencies (MAFs) for our raQTLs as compared to matched SNPs, but only for the K562 dataset and not for the HepG2 dataset (Supplementary Fig. 3c,d). This modest underrepresentation of raQTLs in the human population is consistent with recent computational predictions 13 and may point to a slight negative selection pressure. Taken together, we found no evidence for strong negative selective pressure on raQTLs. 


\section{Integration of SURE with eQTL maps}

Next, we integrated our SuRE data with eQTL mapping data from the GTEx Project8. We compared SuRE data from K562 and HepG2 cells with eQTL data from the most closely related tissues, i.e. whole blood and liver, respectively. Strong similarity between the two data types should not be expected, because in the GTEx data each gene with significant associations (eGene) is linked to $101 \mathrm{eQTL}$ SNPs on average, of which only one or a few may be causal. Rather, we regard the SuRE data as a filter to identify the most likely causal SNPs among the large number of eQTL candidates.

For each raQTL, the $\log _{2}$ (ALT/REF) SuRE signal may be concordant with the eQTL signal (i.e., having the same sign as the slope of the eQTL analysis) or discordant (having the opposite sign). The simplest interpretation of concordance is that the SNP alters a regulatory element that positively regulates the eGene; if an allele reduces the activity of the element then it will also reduce the activity of the eGene. Discordance may point to more indirect mechanisms, e.g., when a SNP alters the promoter of an antisense transcript that in turn interferes with the sense expression of the eGene. In line with this interpretation, concordant raQTLs are enriched near the TSSs of the eGenes (Supplementary Fig. 4a, b). The slightly higher odds ratios for HepG2 vs. liver may be due to a stronger similarity of HepG2 to liver cells than of K562 to blood cells. Because discordant effects are more difficult to interpret, we further focused on SNPs with concordant effects.

\section{Candidate causal SNPs in eQTL maps and their putative mechanism}

We highlight for several physiologically relevant eGenes the most likely causal SNPs based on our SuRE data, and we provide insights into the potential underlying mechanisms.

A first example is $X P N P E P 2$, a gene associated with the risk of angioedema in patients treated with an ACE inhibitor34. The GTEx project has linked the expression of this gene in liver to 33 eQTL SNPs, of which 30 are covered by SuRE (Fig. 4a). Of these, a single SNP (rs3788853) located $\sim 2 \mathrm{~kb}$ upstream of the TSS stands out by a strong ( $\sim 5$-fold difference between the two alleles) and concordant effect on SuRE activity in HepG2 cells (Fig. 4a). This SNP has previously been demonstrated to alter the activity of an enhancer that controls XPNPEP234. Our independent identification of this SNP indicates that SuRE can successfully pinpoint a functionally relevant SNP among a set of eQTL SNPs.

A second example is the $A B C C 11$ gene, which encodes a transmembrane transporter of bile acids, conjugated steroids, and cyclic nucleotides35. GTEx data from liver identified 281 eQTL SNPs surrounding the $A B C C 11$ TSS (Fig. 4b). SuRE data covered 219 (77.9\%) of these, and identified as most likely candidates a cluster of three SNPs within a $\sim 200 \mathrm{bp}$ region located in an intron of $A B C C 11$ (Fig. 4c). Of these, rs11866312 is a likely candidate because the $\mathrm{C}$ allele is predicted to disrupt a binding motif for FOXA1 (Fig. 4d), a pioneer TF that is highly expressed in liver but not in blood8. Indeed, virtually no SuRE effect of these SNPs is observed in K562 cells (data not shown).

A third example are the neighboring genes YEATS4 (encoding a transcription regulator) and $L Y Z$ (encoding lysozyme, an antibacterial protein). Overlapping sets of eQTL SNPs were identified for these genes in whole blood (Fig. 4e and S4c). Among these, SuRE in K562 
cells identified two neighboring raQTLs (rs623853 and rs554591) located $\sim 400$ bp downstream of the YEATS4 TSS, which both show concordance with the eQTL data (Fig. $4 \mathrm{e})$.

To identify TFs that might be responsible for the differential SuRE activity at these two raQTLs, we conducted an in vitro binding proteomic analysis 36,37 . Briefly, we immobilized double-stranded oligonucleotides carrying each of the two alleles on beads, and incubated them with nuclear extract from K562 cells. After washing to remove unbound proteins, we used on-bead trypsin digestion followed by di-methyl stable isotope labeling38 and quantitative mass-spectrometry to identify proteins that preferentially associated with one of the two SNP alleles. In this assay, at rs623853 the weaker A allele caused strong loss of binding of Ets-like factors (ELF) (Fig. 4f), consistent with a disruption of the cognate motif (Fig. 4g). The A allele also showed moderately increased binding of several other proteins. At rs554591 the C allele caused strong loss of ZNF787 and gain of several other factors including KLF and SP proteins (Supplementary Fig. 4d). The variants of both SNPs may thus cause altered binding of TFs and their co-factors, leading to altered enhancer activity. K562 cells are heterozygous for both rs623853 and rs554591, but no significantly different DHS signal is detectable for either of the alleles (Supplementary Fig. 4e,f).

These examples illustrate that SuRE can prioritize SNPs as likely causal candidates from a set of tens to hundreds of eQTL SNP candidates. By integrating our data with the GTEx datasets, SuRE identified at least one raQTL among the eQTL SNPs for $20.0 \%$ of the 8,661 eGenes in whole blood, and for $11.1 \%$ of the 4,000 eGenes in liver.

\section{Integrating SuRE data with GWAS data}

SuRE may also help to identify candidate causal SNPs in GWAS data. We focused on a large GWAS that identified 6,736 lead SNPs and more than 1 million linked significant SNPs associated with at least one of 36 blood-related traits39. These SNPs occurred in LD clusters, each on average consisting of 158 SNPs and represented by a single statistically most significant (lead) SNP. The lead SNPs are not necessarily the causal SNPs, but are more likely to be the causal SNPs or in strong LD with the causal SNP(s). We therefore searched within a $100 \mathrm{~kb}$ window from each lead SNP for overlap between significant GWAS SNPs and raQTLs. For 1,238 out of 6,736 lead SNPs this yielded at least one linked raQTL. These raQTLs were preferentially located close to the lead SNPs, compared to a set of matching control SNPs (Fig. 5a). Overall, the enrichment of SuRE raQTLs among the total set of GWAS SNPs did not significantly exceed that of the matching control set of SNPs (1188), but this was to be expected considering that only one or a few of the on average 158 significant SNPs may be true causal SNPs.

One example where SuRE provided a clear candidate among the GWAS SNPs is rs4572196, which is within $100 \mathrm{~kb}$ of 11 lead SNPs associated with various mature red blood cell traits, such as 'Hemoglobin concentration' and 'Hematocrit'39. In none of the 11 GWAS associations rs4572196 is the lead SNP, but in SuRE the G allele shows an $\sim 8$-fold higher activity than the A allele $\left(P=2.0 \times 10^{-8}\right)$, and it is the only SNP in the region with a $P$ value below our cutoff (Fig. 5b). By in vitro proteomics we identified several proteins with differential binding activity to the two rs 4572196 alleles. JUN proteins showed stronger 
binding to the $\mathrm{G}$ allele (Fig. 5c), as one might predict based on the JUN binding motif (Fig. 5d). The GWAS demonstrated a positive association between the reference A allele and hemoglobin concentration and red blood cell counts39. Interestingly, rs4572196 lies $\sim 11 \mathrm{~kb}$ upstream of $S H 2 B 3 / L N K$, a gene that inhibits erythropoiesis in mouse40. SuRE identifies the A allele as the weak allele, potentially reducing $S H 2 B 3$ expression. We cannot rule out that other SNPs in the region, for example among those not included in our SuRE data, also play a causal role.

Another example is rs3748136, which, together with 66 other SNPs in this locus, was found to be linked to blood counts of reticulocytes. Amongst the 59 SNPs covered in our data, rs3748136 is the only significant SuRE hit, with the A allele showing an $\sim 18$-fold higher activity than the G allele $\left(P=7.5 \times 10^{-20}\right)$ (Fig. 5e). K562 are heterozygous for this SNP and indeed show a strong allelic imbalance in DHS-seq, with the A allele being the more active allele (Fig. 5f). In vitro binding proteomic analysis identified JUN and BACH1 proteins as more strongly bound to the A allele (Fig. $5 \mathrm{~g}$ ), consistent with the $\mathrm{G}$ allele disrupting predicted binding motifs for both proteins (Fig. 5 h). Both BACH1 and JUN proteins are highly expressed in whole blood and in K562 cells8,28. Reanalysis of ChIP data for BACH128 showed a complete allelic imbalance for BACH1 binding and the same was found for JUND (Fig. 5i,j). eQTL analysis of whole blood8 has linked the A allele of rs3748136 to elevated expression of the nearby non-coding RNA gene $N R \_125431$ (Fig. 5k). To further test this, we modified the G allele in K562 cells to an A allele by CRISPR/Cas9 editing41. We found that expression of $N R \_125431$ in K562 cells shows considerable clone to clone variation (Supplementary Fig. 5a-c). To account for this we performed $\mathrm{G}$ to A substitution in a stable K562 clone. This modification led to a 4-fold upregulation of NR_125431 (Fig. 5l).

Finally, we overlaid the HepG2 SuRE data with a recent GWAS that linked SNPs to the risk of hepatitis B virus (HBV) related hepatocellular carcinoma (HCC), a type of cancer prevalent in Asia42. This study identified 61 candidate SNPs in a $\sim 200 \mathrm{~kb}$ region that includes the HLA class 1 locus. Of the 50 SNPs covered by our SuRE data, two are raQTLs in HepG2 (Fig. 6). These raQTLs are intergenic; both ALT alleles show reduced SuRE signals and are associated with higher HCC risk. Besides the $H L A$ genes that are important for antigen presentation, other genes in this region could be affected by these raQTLs and play a role in HCC. For example, $Z N R D 1$ and its antisense transcript have been implicated in expression of HBV mRNA and proliferation of HBV-infected HepG2 cells43.

\section{Discussion}

By surveying 5.9 million SNPs from four entire human genomes we identified about 30,000 SNPs that alter regulatory activity of enhancers or promoters. The data are available for download, and can be queried through a web application (https://sure.nki.nl).

Because $90 \%$ of these raQTLs were identified in only one of the two tested cell lines, it is likely that extension of this survey to other cell types will increase the number of raQTLs substantially. It is thus conceivable that several percent of all human SNPs may have impact on the activity of regulatory elements in at least one cell type. 
While the redundant design of SuRE increases the odds that a robust and biologically representative measure of SNP effects is obtained, we note that SuRE signals arising from enhancers are generally weaker than those from promoters, and it cannot be ruled out that certain enhancers cannot be detected by SuRE at all. Thus, our approach may be better powered to detect effects of SNPs on promoters compared to enhancers. Furthermore, SuRE infers effects of SNPs on enhancer activity indirectly, by virtue of the ability of most enhancers to act as autonomous TSS. Although this feature generally correlates with the potency of enhancers to activate a cis-linked promoter, this correlation is not perfect 44 and in some enhancers both activities may be differentially affected by particular SNPs.

Like most other MPRAs, SuRE queries all DNA elements in a plasmid context and in cultured cell lines, which may yield different results compared to a proper genomic context and tissue context. Integration with multiple orthogonal datasets can help provide confidence in the relevance of candidate SNPs. Epigenomic data, sequence motif analysis and in vitro binding mass-spectrometry 36,37 can serve this purpose, and in addition provide key insights into the mechanisms of action.

We foresee several additional applications of these SuRE data. First, there are many other eQTL studies and GWAS that may be overlaid with the SuRE maps. Second, in addition to SNPs, small insertions and deletions (indels) may be analyzed. While in human genomes such indels occur at a $\sim 20$ fold lower frequency than SNPs1, their individual regulatory impact may be more potent, as they tend to disrupt TF binding motifs more dramatically. Third, our datasets may be useful for studying the regulatory grammar of TFs, as they cover natural genetic variation in thousands of regulatory elements. For example, the SuRE data may be used to refine computational predictions of SNP effects13,30,45. Finally, it will be interesting to expand this type of analysis to individuals with a genetic disorder to capture additional disease-relevant variants that might not be found in the general population.

\section{Online Methods}

\section{SuRE library preparation and barcode-to-fragment association}

SuRE libraries were generated as described previously26. DNA was isolated from lymphoblast cell lines HG02601, GM18983, HG01241 and HG03464 obtained from Coriell Institute, fragmented and gel-purified to obtain $~ 300$-bp elements. For each genome, two SuRE libraries were generated, each of an approximate complexity of 300 million fragmentbarcode pairs. This was done by transformation in CloneCatcher DH5G electrocompetent Escherichia coli cells (\#C810111; Genlantis), or in E. cloni 10G (\#60107-1; Lucigen).

Barcode-to-fragment association was done as described previously26, except that because of the smaller genomic insert size no digest with a frequent cutter was required. Thus, after ICeuI digest and self-ligation we immediately proceeded to the I-SceI digest.

\section{Cell culture and transfection for SuRE}

K562 cells were cultured and transfected as described26. HepG2 (\#HB 8065; ATCC) were cultured according to supplier's protocol and transiently transfected in the same manner as K562 cells except that program T-028 was used for nucleofection, $7.5 \mu \mathrm{g}$ plasmid was used 
for each 5 million cells, and cells were harvested 48 hours after transfection. One hundred million cells were transfected for each replicate. Every 3 months all cells in culture were screened for mycoplasma using PCR (\#6601; Takara).

\section{Illumina sequencing}

Paired end sequencing (150 bp) of SuRE libraries was done by Novogene on the HiSeq-X platform, generating about 1 billion reads per library. Standard full genome sequencing and allele calling for the K562 cell line was done by Novogene on the HiSeq-X10 platform with PE 150 -bp reads amounting to approximately $100 \mathrm{~Gb}$ or a $\sim 30$ fold coverage of the genome. Single-end sequencing on reverse transcribed, PCR-amplified barcodes was done by the NKI Genomics Core Facility on a HiSeq2500 machine.

\section{Sequencing data processing}

Paired-end reads (PE reads) of the SuRE libraries (for associating genomic positions and barcodes for each SuRE-fragment), and single-end reads (SE reads) of the PCR amplified barcodes (representing raw SuRE expression data), were processed to remove adapter and vector backbone sequences, using cutadapt(V1.9.1)46. PE and SE reads were discarded if the barcode sequence contained Ns or the sequence was not exactly 20 nucleotides. The remaining sequences in the PE reads are combinations of barcode sequences and genomic DNA (gDNA) sequences, whereas the SE reads only yield barcode sequences. The latter barcodes are simply recorded and counted. The gDNA sequences of the SuRE libraries were mapped to the reference genome sequence (hg19, including only chr1-22, chrX), using bowtie2 (V2.3.2,47), with a maximum insert length set to $1 \mathrm{~kb}$. Read pairs with either the forward or the reverse gDNA sequence less than 6 nucleotides, and read pairs not aligned as 'proper pair', were discarded. To prevent allelic biases in alignment we used WASP48 and SNP annotations from the 1000-genome project (external data source \#17) to discard all reads potentially resulting in biased alignments.

The resulting associations of barcode sequence-genomic position pairs were further processed as follows:

1. Identical barcodes associated with multiple alignment positions were discarded except for the most abundant barcode-position pair.

2. Different barcodes associated with the exact same alignment position were merged; i.e. the barcode sequence associated with this position was set to the most frequent barcode sequence in the set, and the total number of PE reads in the set was used as count for this barcode-position pair.

Next, the barcodes identified in the SE reads were matched to the barcodes in the remaining barcode-position pairs, and 'SuRE-count'tables were generated associating barcode sequences, genomic positions, and counts for associated PE reads and matched SE reads for each of the biological replicates.

\section{SNP annotation}

The fragments, specified in the SuRE-count tables were further annotated with SNP positions and base identities. For this annotation only SNPs were considered which were 
single-nucleotide, bi-allelic, and the alternative allele in at least 1 of all 4 considered genomes. SNPs homozygous for the reference allele in all 4 genomes were discarded. For each SNP in such a fragment we determined its base identity as observed in the actual sequence reads. Some fragments are too long to be entirely covered by the PE reads. In these cases the unidentified SNPs were assigned the IUPAC representation of both alleles; if the two alleles were known to be identical in the genome that was used to construct the particular library (based on annotation by the 1000 genome project, external data source \#17), this inferred allele was used for annotation. At the time of finalizing this article we noticed that, due to a minor coding error, $\sim 0.4 \%$ of the SuRE fragments was incorrectly annotated to carry both the REF and ALT allele of a SNP. This may cause a very slight underestimate of the total number of raQTLs, but it does not not alter any of the reported conclusions.

\section{SURE data analysis and visualization}

Data analysis and figure production was mostly done using various R (https://www.Rproject.org) and BioConductor49 packages.

\section{Generating BigWigs of SuRE enrichment profiles}

For each strand separately, we determined the cDNA barcode count for all SuRE fragments overlapping a given position. This total was divided by the total counts of the SuRE fragments measured in the SuRE library (iPCR barcode counts) to give the SuRE enrichment score. Within each transfection replicate the genome-wide normalized SuRE enrichment score was scaled to a mean of 1 . Transfection replicates were then averaged to yield a genome-wide normalized SuRE enrichment profile per SuRE library. Then, the library replicates were also averaged to yield a genome-wide normalized SuRE enrichment profile per genome. Of these datasets BigWig files were generated. This analysis was done disregarding SNPs and is therefore independent of the identification of the raQTLs (see below).

\section{Identification of raQTLs}

1 Equalization of cDNA barcode sequencing depth-To minimize biases that might be caused by excessive differences in sequencing depth, cDNA reads of some samples were sub-sampled. First, for each transfection replicate the relative sequencing depth of cDNA barcodes was determined as the total cDNA barcode counts divided by the corresponding library complexity (i.e. the number of unique fragments identified in the library). Then, samples with a relative cDNA sequencing depth that exceeded the mean of all samples by more than one standard deviation (i.e., all K562 and HepG2 transfection replicates for genome HG02601 library 1, and all HepG2 transfection replicates for genome HG02601 library 2) were down-sampled to the mean relative cDNA read depth.

2 SURE signal normalization per fragment-For each fragment in each of the 8 SuRE libraries we normalized the iPCR barcode count by dividing it to the total iPCR sequencing depth of that library, expressed as reads per billion. Similarly, we normalized cDNA barcode counts per transfection replicate by dividing it by the total cDNA sequencing depth, again expressed as reads per billion. Next, for each fragment we calculated the SuRE 
signal $\left(\mathrm{S}_{\mathrm{SuRE}}\right)$, which is the mean of all transfection replicates of the ratio of the normalized cDNA barcode count over normalized iPCR barcode count. Since both values used to obtain this ratio are expressed as reads per billion, we interpret the resulting $\mathrm{S}_{\mathrm{SuRE}}$ values as an enrichment score.

\section{Aggregation of SuRE signals by SNP allele and $P$ value calculation-Using} this $\mathrm{S}_{\text {SuRE }}$ per fragment, a mean $\bar{S}_{S U R E}$ was calculated for each allele of each SNP as the mean $\mathrm{S}_{\mathrm{SuRE}}$ of all fragments containing that SNP allele. Also, for each SNP a two-sided Wilcoxon rank-sum test was performed comparing the set of $\mathrm{S}_{\text {SuRE }}$ values of all fragments containing the REF allele with the set of $\mathrm{S}_{\mathrm{SuRE}}$ values of all fragments containing the ALT allele. The resulting vector of $P$ values (one for each SNP) is referred to as $P_{t}$. In addition, to estimate the False Discovery Rate (FDR), the same two-sided Wilcoxon rank-sum test was applied once after random shuffling of the $\mathrm{S}_{\mathrm{SuRE}}$ of the fragments among the two alleles, yielding a vector of random $P$ values referred to as $P_{r}$.

4 Criteria for raQTL definition-We then focused on those SNPs for which both alleles were covered by at least 10 fragments and no more than 999 fragments, and for which at least one of the alleles met the criterium $\bar{S}_{S u R E}>4$ (170,118 SNPs in K562; 395,756 SNPs in HepG2). For FDR $=5 \%$, we then chose the lowest $P$ value cutoff $\mathrm{p}_{\text {cut }}$ for which the number of SNPs with $P_{t}<\mathrm{p}_{\text {cut }}$ was at least 20 times larger than the number of SNPs with $P_{r}<\mathrm{p}_{\text {cut }}$. We refer to this set of SNPs as raQTLs. The same procedure was applied separately for data from K562 and HepG2 cells. For K562 $\mathrm{p}_{\text {cut }}$ was 0.006192715 and for HepG2 $\mathrm{p}_{\text {cut }}$ was 0.00173121 .

\section{Enrichment of raQTLs in ENCODE classes}

For Figure 2a we used the GenomicRanges package of BioConductor49 to determine the enrichment or depletion of raQTLs in ENCODE chromatin classes (external dataset \#7) as compared to all 5.9 million SNPs we assessed.

\section{Comparison of SuRE to DNase-seq, H3K27ac and ATAC-seq allelic imbalance}

In Figure 2 we plotted the DNase-seq signal around the raQTLs using external data source \#4 and BioConductor package CoverageView (version 1.4.0) and 25-bp windows. Figure 2c was generated from the same data. For the analysis of allelic imbalance in the DNase-seq signal we combined three available experiments (external data source \#1,\#2,\#3) and extracted from the bam-files the reads that overlapped a SNP that was found to be heterozygous in K562 in our own genome sequencing analysis (see 'Illumina sequencing' section). We focused on those raQTLs for which we found at least 20 DNase-seq reads and quantified the ratio of reads containing the REF allele over the reads containing the ALT allele, after adding for each allele a pseudocount of 1. Similarly, from our own genome sequencing of K562 we quantified the ratio of reads containing the REF allele over reads containing the ALT allele, after adding for each allele a pseudocount of 1. Finally, the DNase allelic imbalance was calculated as the DNase-seq allele-ratio over the genomic allele-ratio. 
For the SuRE data, the allelic imbalance was calculated as the ratio of $\mathrm{S}_{\mathrm{SuRE}}$ for the REF allele over the $\mathrm{S}_{\mathrm{SuRE}}$ of the ALT allele (since both these values are already normalized for coverage in the libraries).

To obtain a matching set of control SNPs we intersected a DNase-peak annotation (external data source \#7) with our SNPs, and we retrieved the DNase-seq allele counts for the 2,500 overlapping SNPs with the highest SuRE $P_{t}$ values. We required at least DNase-seq 20 reads covering the SNP, and from the resulting set we randomly selected a subset of SNPs of the same size as the set of raQTLs (Fig. 2f). To this control set we applied the same analysis of allelic imbalance as to the raQTLs. The comparison in Supplementary Figure 2 with H3K27ac (external data source \#20,21) and ATAC-seq (external data source \#22,23) we did in the same manner as described for the DNase-seq data except that we required only 10 reads to cover the SNP, since these data are of approximately 5-10 times lower sequencing depth. We excluded a third ATAC-seq replicate (GSM2695562) since for this replicate we did not observe a similar pattern of enrichment as for the other ATAC-seq replicates. The mean enrichment profiles shown in Supplementary Figure 2c-e were generate with BioConductor package CoverageView (version 1.4.0).

\section{Re-mapping of BACH1 and JUND ChIP-seq data}

Fastq files were downloaded from the SRA repository (external dataset \#13, \#14) using fastq-dump from the SRA-tools package. For BACH1 we downloaded data from datasets SRR502556 and SRR502557, for JUND from datasets SRR502542 and SRR502543. Reads were aligned to the human reference sequence (hg19, including only chr1-22, chrX), using bowtie2 (V2.3.2) 47 with default settings.

\section{Allele frequencies}

MAFs of SNPs were obtained from the 1000 Genomes Project (external data source \#17). Common SNPs were defined as SNPs with MAF > 0.05. Of the 5,919,293 SNPs in our SuRE dataset $4,569,323$ classify as common. This is $\sim 57 \%$ of the estimated $\sim 8$ million common SNPs according to the 1000 Genomes Project1. The proportion of raQTLs for which the SuRE effect could be resolved to a single SNP was calculated as the fraction of raQTLs for which neither neighbor SNP was also a raQTL.

\section{TF motif analysis}

We made use of SNP2TFBS (external data source \#16)30 to identify all SNPs for which there was a difference in predicted affinity for a TF between the REF and ALT allele. For each SNP we only considered the TF listed first in SNP2TFBS (i.e., the TF with the biggest absolute difference in motif score). For the comparison of motif disruptions in K562 and HepG2 we identified all raQTLs of K562 and HepG2 that caused motif disruptions, we down-sampled the K562 disruptions to the same number as the HepG2 disruptions (since K562 has more raQTLs), and plotted the ratio of the counts (plus one pseudo-count) for the 7 most extreme ratios for each cell type. Sequence logos were obtained from external dataset $\# 19$. 


\section{Compiling a set of control SNPs that are matched to the significant SNPs}

For the analyses in Supplementary Figure 3 and Figure 5a we compared the set of raQTLs to a control set of matching SNPs that was selected as follows. We ranked all SNPs, first: by the $S_{\text {SuRE }}$ (rounded to whole numbers) of the strongest allele, second: by the number of fragments containing the least covered allele, and third: by the number of fragments containing the most covered allele. This is intended to rank them based on regulatory element activity $\left(\mathrm{S}_{\mathrm{SuRE}}\right)$ and our sensitivity to detect a significant difference (coverage of the alleles). Then we identified the raQTLs along this ranking and selected both direct neighbors, removed the raQTLs and down-sampled the resulting set to yield the same number of matched SNPs as raQTLs.

\section{raQTL density around loss-of-function tolerant and loss-off-function intolerant genes}

For the definition of LOF-tolerant and LOF-intolerant genes, we used external dataset \#11 and the same cut-offs as previously reported33, classifying genes as having pLI scores of > 0.9 as intolerant and $<0.1$ as tolerant. We then matched these genes to GENCODE version 19 annotation (external data source \#12) to identify the corresponding TSSs. Using the GenomicRanges package of BioConductor49 we defined regions around these TSSs of $\pm 100 \mathrm{~kb}$ and subtracted all possible exons (as defined in GENCODE version 19). In the resulting regions we then determined the fraction of all SNPs that is a raQTL, and the fraction that belonged to the set of matched control SNPs. This analysis was done for both LOF-intolerant and LOF-tolerant genes.

\section{Integration with eQTL data}

GTEx eQTL data8 (release v7; external data source \#18) were downloaded on 27 January 2018. For whole blood we used the extracted file

Whole_Blood.v7.signif_variant_gene_pairs.txt.gz and for liver we used Liver.v7.signif_variant_gene_pairs.txt.gz.

Gene annotation tracks in Figure 4 were generated by the Gviz package of BioConductor, function BiomartGeneRegionTrack(). ENCODE28 DNase-seq data used in this figure are from external data source \#4, \#15.

\section{Integration with GWAS data}

Overlap between SNPs identified in the GWAS study by Astle et al.39, was obtained by searching for significant SuRE SNPs within $100 \mathrm{~kb}$ of each of the 6,736 lead SNPs identifying 1,238 lead SNPs within $100 \mathrm{~kb}$ of at least one significant SuRE SNP (external data source \#6). The window of $100 \mathrm{~kb}$ was chosen to be substantially larger than the typical size of an LD block. For these lead SNPs we calculated the distance to SNP with the lowest $P$ value in our SuRE data (Fig. 5a). As a control we did the same procedure for the set of matched SuRE SNPs. In Figure 5 only those SNPs with significant $P$ values in the GWAS (cutoff: $P<8.31 \times 10^{-9}$; ref. 39 ) are shown. Note that what we refer to as 'lead SNPs' are called 'conditionally independent index-variant associations' in the original GWAS39. Gene annotation tracks in Figure 5 were generated by the Gviz package of BioConductor, function BiomartGeneRegionTrack(). DNase-seq data used are from external data source \#4. 


\section{CRISPR/Cas9-mediated editing of rs3748136}

We performed our CRISPR experiments on a K562 subclone in which NR_125431 was active (subclone BL_2) because initial experiments revealed that in the $\mathrm{K} 562$ pool, $N R \_125431$ is expressed in only $\sim 25 \%$ of the cells (Supplementary Fig. 5c). Five million of the BL_2 cells were nucleofected as described above with $2 \mu \mathrm{g}$ of vector pX330-U6Chimeric_BB-CBh-hSpCas9, a gift from Feng Zhang (Addgene plasmid \# 4223041) encoding Cas9 and the chimeric guide RNA; and 20 pmol repair template (see Supplementary Table 2 for nucleotide sequences of guide RNAs). Cells were then cultured for 3 days in the presence of $1 \mu \mathrm{M}$ DNA-PK inhibitor (\#NU7441; Cayman Chemical Company) and then expanded for another 5 days without this inhibitor. For genotyping we used a PCR amplicon that that included SNP rs453301, 250 bp downstream of rs3748136 that was also heterozygous in K562. After confirming editing efficiency for the population of cells using Sanger sequencing and TIDE analysis50, single cells were cloned out. After expansion, clones were genotyped on the single PCR amplicon, and classified as successfully edited when they were heterozygous (i.e. not edited) at rs453301 but homozygous for rs3748136, or they were classified as wild-type when both loci were still heterozygous. Of note, we identified many clones in which our CRISPR editing caused deletions around the targeted SNP; these were discarded. Successfully edited clones and wild-type clones were then analyzed for RNA expression by RT-qPCR. Since the chromosome that was edited at rs3748136 was the only chromosome showing expression of $N R \_125431$ to begin with, we are looking at the increased expression from that chromosome after editing (see main text), even though the RT-qPCR is not allele specific. See Supplementary Table 2 for oligonucleotide sequences used.

\section{Targeted Locus Analysis}

In order to determine the phasing of the K562 genome around rs1053036, TLA was performed essentially as described51. Briefly, roughly 5 million K562 cells were crosslinked with $4 \%$ formaldehyde and cut with NlaIII. After ligation, the template was decrosslinked and further digested with NspI. The second ligation yields circular DNA that is used as input for the inverse PCR reaction. We performed two PCR reactions: the first with primers adjacent to rs 1053036, located in the last exon of the $N R_{-} 125431$ and the second with primers adjacent to rs3748136, located in the intergenic region (Supplementary Table 2). The PCR amplicons were combined and we generated sequencing libraries using the KAPA High Throughput Library Preparation Kit (\# 7961901001; Roche). We generated $2 \times$ 150-bp PE sequences on an Illumina MiSeq. Sequence reads were mapped to hg19 using BWA-SW52. The resulting bam files and the K562 vcf file (obtained from whole genome sequencing at Novogene) were used as input for HapCUT253 with the --hic option turned on to phase the alleles.

\section{RT-qPCR}

RNA was isolated from 1-5 million cells using Trisure (\#BOI-38033; Bioline). DNase digestion was performed on $1.5 \mu \mathrm{g}$ RNA with 10 units DNase I for $30 \mathrm{~min}$ (\#04716728001; Roche) and DNase I was inactivated by addition of $1 \mu \mathrm{l} 25 \mathrm{mM}$ EDTA and incubation at $70{ }^{\circ} \mathrm{C}$ for $10 \mathrm{~min}$. cDNA was produced by adding $1 \mu \mathrm{l} 50 \mathrm{ng} / \mu \mathrm{l}$ random 
hexamers and $1 \mu \mathrm{dNTPs}\left(10 \mathrm{mM}\right.$ each) and incubated for 5 minutes at $65^{\circ} \mathrm{C}$. Then $4 \mu \mathrm{l}$ of first strand buffer, 20 units RNase inhibitor (\#EO0381; ThermoFisher Scientific), $1 \mu$ of Tetro reverse transcriptase (\#BIO-65050; Bioline) and $2 \mu \mathrm{l}$ water was added and the reaction mix was incubated for 10 minutes at $25^{\circ} \mathrm{C}$ followed by 45 minutes at $45^{\circ} \mathrm{C}$ and heatinactivation at $85^{\circ}$ for 5 minutes. qPCR was performed on the Roche LightCycler480 II using the Sensifast SYBR No-ROX mix (\#BIO-98020; Bioline). All expression levels were calculated using the $2^{-\triangle \Delta \mathrm{Ct}}$ method and normalized to the internal control GAPDH. See Supplementary Table 2 for oligonucleotide sequences used for qPCR.

\section{DNA affinity purification and LC-MS analysis}

Nuclear extracts were generated from K562 cells essentially as described54. Briefly, cells were washed with PBS and then resuspended in $5 \times$ cell pellet volumes of hypotonic Buffer A (10 mM HEPES (pH 7.9), $1.5 \mathrm{mM} \mathrm{MgCl}_{2}, 10 \mathrm{mM} \mathrm{KCl).} \mathrm{After} \mathrm{incubation} \mathrm{for} 10$ minutes at $4{ }^{\circ} \mathrm{C}$, cells were collected by centrifugation and resuspended in two pellet volumes of buffer A supplemented with $0.15 \%$ NP40. Cells were then lysed by dounce homogenization using 35 strokes with a type B (tight) pestle on ice. Crude nuclei were collected by centrifugation and then lysed in two pellet volumes of Buffer C $(420 \mathrm{mM} \mathrm{NaCl}, 20 \mathrm{mM}$ HEPES (pH 7.9), $20 \%$ (v/v) glycerol, $2 \mathrm{mM} \mathrm{MgCl}_{2}, 0.2 \mathrm{mM}$ EDTA, $0.1 \%$ NP40, EDTAfree complete protease inhibitors (Roche), and $0.5 \mathrm{mM} \mathrm{DTT}$ ) by rotation for $1 \mathrm{~h}$ at $4{ }^{\circ} \mathrm{C}$. After centrifugation for 20 minutes at $21,000 \mathrm{~g}$, nuclear extract was collected as the soluble fraction. This extract was then aliquoted, snap-frozen and stored at $-80{ }^{\circ} \mathrm{C}$ until further usage.

Oligonucleotides for the DNA affinity purifications were ordered from Integrated DNA Technologies with the forward strand containing a 5' biotin moiety; see Supplementary Table 2). DNA affinity purifications and on-bead trypsin digestion was performed on 96well filter plates essentially as described37. Tryptic peptides from SNP allele pull-downs were desalted using Stage (stop and go extraction) tips and then subjected to stable isotope di-methyl labeling on the Stage tips38. Matching light and heavy peptides were then combined and samples were finally subjected to LC-MS and subsequent data analyses using MaxQuant55 and R essentially as described36.

\section{Data availability statement}

Raw SuRE sequencing data are available at GEO (https://www.ncbi.nlm.nih.gov/geo/) under accession GSE128325. SuRE count tables, BigWig files for visualization of SuRE data tracks in genome browsers, lists of raQTLs and a table with SuRE data for all 5.9 million SNPs are available from the Open Science Framework: https://osf.io/w5bzq/wiki/home/? view. SuRE data can also be queried and visualized at https://sure.nki.nl. URLs to external data sources are listed in Supplementary Table 3.

\section{Statistics}

For the identification of raQTLs we tested the difference in SuRE expression of fragments containing the reference allele and the fragments containing the alternative allele using a two-sided Wilcoxon rank-sum test (see also section 'Identification of raQTLs'). In addition, the same two-sided Wilcoxon rank-sum test was applied once after random shuffling of 
fragments among the two alleles. Using all obtained $P$ values from the real comparisons and the shuffled comparisons, we estimated the False Discovery Rate (FDR). In Figure 2a, a two-sided Fisher exact test was performed, yielding $P<2.2 \times 10^{-16}$ for each of the comparisons. The number of raQTLs overlapping with each of these states was CTCF: 667; enhancer: 1,052; promoter flanking: 74; repressed: 11,024; transcribed: 1,414; transcription start site: 2,189; weak enhancer: 576, while the number of all SNPs overlapping was CTCF: 79,437; enhancer: 44,296; promoter flanking: 2,178; repressed: 4,701,204; transcribed: 626,914; transcription start site: 46,631; weak enhancer: 17,665. For comparisons of alleles observed for DNA sequencing and DNase-seq, ChIP-seq or RNA-seq in Figures 2e, 5f, 5i, 5j and Supplementary Figures $4 \mathrm{e}$,f and $5 \mathrm{~b}, P$ values were obtained using a one-side Fisher exact test.

$P$ values in Figure 3b,c were obtained using a two-sided Fisher exact test. For the comparison in Figure 5a, a two-sided Wilcoxon rank-sum test was done to compare the distances to the lead SNP for the raQTLs with the distances of the matched SNPs to the lead SNP.

\section{Code availability statement}

Scripts are available on https://github.com/vansteensellab/SuRE-SNV-code.

Software used is described in the relevant methods section and in the Life Sciences Reporting Summary.

\section{Supplementary Material}

Refer to Web version on PubMed Central for supplementary material.

\section{Acknowledgements}

We thank the NKI Genomics Core Facility and Research IT team for technical support; the RHPC facility of the Netherlands Cancer Institute for providing computational resources; members of our laboratories for helpful discussions. Supported by ERC Advanced Grant 694466 (B.v.S.); ERC Starting Grant 637587 (E.d.W.); NIH grant R01HG003008 and Columbia University's Vagelos Precision Medicine Pilot Program (H.J.B.). F.C. was supported by a Swiss National Science Foundation postdoctoral fellowship (P2EZP3_165206). J.v.A., L.P., M.d.H., M.P.B., F.C., R.v.d.W., H.T., E.d.W., M.V., and B.v.S. are part of the Oncode Institute, which is partly funded by the Dutch Cancer Society (KWF).

\section{References}

1. 1000 Genomes Project Consortium. A global reference for human genetic variation. Nature. 2015; 526:68-74. [PubMed: 26432245]

2. Gusev A, et al. Partitioning heritability of regulatory and cell-type-specific variants across 11 common diseases. Am J Hum Genet. 2014; 95:535-52. [PubMed: 25439723]

3. Albert FW, Kruglyak L. The role of regulatory variation in complex traits and disease. Nat Rev Genet. 2015; 16:197-212. [PubMed: 25707927]

4. Miguel-Escalada I, Pasquali L, Ferrer J. Transcriptional enhancers: functional insights and role in human disease. Curr Opin Genet Dev. 2015; 33:71-6. [PubMed: 26433090]

5. Deplancke B, Alpern D, Gardeux V. The Genetics of Transcription Factor DNA Binding Variation. Cell. 2016; 166:538-554. [PubMed: 27471964]

6. MacArthur J, et al. The new NHGRI-EBI Catalog of published genome-wide association studies (GWAS Catalog). Nucleic Acids Res. 2017; 45:D896-D901. [PubMed: 27899670] 
7. Gallagher MD, Chen-Plotkin AS. The Post-GWAS Era: From Association to Function. Am J Hum Genet. 2018; 102:717-730. [PubMed: 29727686]

8. GTEx Consortium. Genetic effects on gene expression across human tissues. Nature. 2017; 550:204-213. [PubMed: 29022597]

9. Schaid DJ, Chen W, Larson NB. From genome-wide associations to candidate causal variants by statistical fine-mapping. Nat Rev Genet. 2018; 19:491-504. [PubMed: 29844615]

10. Nishizaki SS, Boyle AP. Mining the Unknown: Assigning Function to Noncoding Single Nucleotide Polymorphisms. Trends Genet. 2017; 33:34-45. [PubMed: 27939749]

11. Farh KK, et al. Genetic and epigenetic fine mapping of causal autoimmune disease variants. Nature. 2015; 518:337-43. [PubMed: 25363779]

12. Tak YG, Farnham PJ. Making sense of GWAS: using epigenomics and genome engineering to understand the functional relevance of SNPs in non-coding regions of the human genome. Epigenetics Chromatin. 2015; 8:57. [PubMed: 26719772]

13. Zhou J, et al. Deep learning sequence-based ab initio prediction of variant effects on expression and disease risk. Nat Genet. 2018; 50:1171-1179. [PubMed: 30013180]

14. Degner JF, et al. DNase I sensitivity QTLs are a major determinant of human expression variation. Nature. 2012; 482:390-4. [PubMed: 22307276]

15. McVicker G, et al. Identification of genetic variants that affect histone modifications in human cells. Science. 2013; 342:747-9. [PubMed: 24136359]

16. Kilpinen $\mathrm{H}$, et al. Coordinated effects of sequence variation on DNA binding, chromatin structure, and transcription. Science. 2013; 342:744-7. [PubMed: 24136355]

17. Kasowski M, et al. Extensive variation in chromatin states across humans. Science. 2013; 342:7502. [PubMed: 24136358]

18. Waszak SM, et al. Population Variation and Genetic Control of Modular Chromatin Architecture in Humans. Cell. 2015; 162:1039-50. [PubMed: 26300124]

19. Grubert F, et al. Genetic Control of Chromatin States in Humans Involves Local and Distal Chromosomal Interactions. Cell. 2015; 162:1051-65. [PubMed: 26300125]

20. Gate RE, et al. Genetic determinants of co-accessible chromatin regions in activated $\mathrm{T}$ cells across humans. Nat Genet. 2018; 50:1140-1150. [PubMed: 29988122]

21. Vockley CM, et al. Massively parallel quantification of the regulatory effects of noncoding genetic variation in a human cohort. Genome Res. 2015; 25:1206-14. [PubMed: 26084464]

22. Tewhey R, et al. Direct Identification of Hundreds of Expression-Modulating Variants using a Multiplexed Reporter Assay. Cell. 2016; 165:1519-1529. [PubMed: 27259153]

23. Ulirsch JC, et al. Systematic Functional Dissection of Common Genetic Variation Affecting Red Blood Cell Traits. Cell. 2016; 165:1530-1545. [PubMed: 27259154]

24. Liu S, et al. Systematic identification of regulatory variants associated with cancer risk. Genome Biol. 2017; 18:194. [PubMed: 29061142]

25. Zhang $\mathrm{P}$, et al. High-throughput screening of prostate cancer risk loci by single nucleotide polymorphisms sequencing. Nat Commun. 2018; 9

26. van Arensbergen J, et al. Genome-wide mapping of autonomous promoter activity in human cells. Nat Biotechnol. 2017; 35:145-153. [PubMed: 28024146]

27. Nakamura M, et al. Genome-wide association study identifies TNFSF15 and POU2AF1 as susceptibility loci for primary biliary cirrhosis in the Japanese population. Am J Hum Genet. 2012; 91:721-8. [PubMed: 23000144]

28. Encode Project Consortium. An integrated encyclopedia of DNA elements in the human genome. Nature. 2012; 489:57-74. [PubMed: 22955616]

29. Liu X, et al. In Situ Capture of Chromatin Interactions by Biotinylated dCas9. Cell. 2017; 170:1028-1043 e19. [PubMed: 28841410]

30. Kumar S, Ambrosini G, Bucher P. SNP2TFBS - a database of regulatory SNPs affecting predicted transcription factor binding site affinity. Nucleic Acids Res. 2017; 45:D139-D144. [PubMed: 27899579]

31. Law JC, Ritke MK, Yalowich JC, Leder GH, Ferrell RE. Mutational inactivation of the p53 gene in the human erythroid leukemic K562 cell line. Leuk Res. 1993; 17:1045-50. [PubMed: 8246608] 
32. Westerink WM, Stevenson JC, Horbach GJ, Schoonen WG. The development of RAD51C, Cystatin A, p53 and Nrf2 luciferase-reporter assays in metabolically competent HepG2 cells for the assessment of mechanism-based genotoxicity and of oxidative stress in the early research phase of drug development. Mutat Res. 2010; 696:21-40. [PubMed: 20006733]

33. Lek M, et al. Analysis of protein-coding genetic variation in 60,706 humans. Nature. 2016; 536:285-91. [PubMed: 27535533]

34. Cilia La Corte AL, et al. A functional XPNPEP2 promoter haplotype leads to reduced plasma aminopeptidase $\mathrm{P}$ and increased risk of ACE inhibitor-induced angioedema. Hum Mutat. 2011; 32:1326-31. [PubMed: 21898657]

35. Chen ZS, Guo Y, Belinsky MG, Kotova E, Kruh GD. Transport of bile acids, sulfated steroids, estradiol 17-beta-D-glucuronide, and leukotriene $\mathrm{C} 4$ by human multidrug resistance protein 8 (ABCC11). Mol Pharmacol. 2005; 67:545-57. [PubMed: 15537867]

36. Makowski MM, et al. An interaction proteomics survey of transcription factor binding at recurrent TERT promoter mutations. Proteomics. 2016; 16:417-26. [PubMed: 26553150]

37. Makowski MM, et al. Global profiling of protein-DNA and protein-nucleosome binding affinities using quantitative mass spectrometry. Nat Commun. 2018; 9

38. Boersema PJ, Raijmakers R, Lemeer S, Mohammed S, Heck AJ. Multiplex peptide stable isotope dimethyl labeling for quantitative proteomics. Nat Protoc. 2009; 4:484-94. [PubMed: 19300442]

39. Astle WJ, et al. The Allelic Landscape of Human Blood Cell Trait Variation and Links to Common Complex Disease. Cell. 2016; 167:1415-1429 e19. [PubMed: 27863252]

40. Maslah N, Cassinat B, Verger E, Kiladjian JJ, Velazquez L. The role of LNK/SH2B3 genetic alterations in myeloproliferative neoplasms and other hematological disorders. Leukemia. 2017; 31:1661-1670. [PubMed: 28484264]

41. Cong L, et al. Multiplex genome engineering using CRISPR/Cas systems. Science. 2013; 339:81923. [PubMed: 23287718]

42. Sawai H, et al. Genome-wide association study identified new susceptible genetic variants in HLA class I region for hepatitis B virus-related hepatocellular carcinoma. Sci Rep. 2018; 8

43. Wen J, et al. Expression quantitative trait loci in long non-coding RNA ZNRD1-AS1 influence both HBV infection and hepatocellular carcinoma development. Mol Carcinog. 2015; 54:1275-82. [PubMed: 25110835]

44. Nguyen TA, et al. High-throughput functional comparison of promoter and enhancer activities. Genome Res. 2016; 26:1023-33. [PubMed: 27311442]

45. Ward LD, Kellis M. HaploReg v4: systematic mining of putative causal variants, cell types, regulators and target genes for human complex traits and disease. Nucleic Acids Res. 2016; 44:D877-81. [PubMed: 26657631]

46. Martin M. Cutadapt removes adapter sequences from high-throughput sequencing reads. 2011; 17:3.

47. Langmead B, Salzberg SL. Fast gapped-read alignment with Bowtie 2. Nat Methods. 2012; 9:3579. [PubMed: 22388286]

48. van de Geijn B, McVicker G, Gilad Y, Pritchard JK. WASP: allele-specific software for robust molecular quantitative trait locus discovery. Nat Methods. 2015; 12:1061-3. [PubMed: 26366987]

49. Huber W, et al. Orchestrating high-throughput genomic analysis with Bioconductor. Nat Methods. 2015; 12:115-21. [PubMed: 25633503]

50. Brinkman EK, Chen T, Amendola M, van Steensel B. Easy quantitative assessment of genome editing by sequence trace decomposition. Nucleic Acids Res. 2014; 42:e168. [PubMed: 25300484]

51. de Vree PJ, et al. Targeted sequencing by proximity ligation for comprehensive variant detection and local haplotyping. Nat Biotechnol. 2014; 32:1019-25. [PubMed: 25129690]

52. Li H, Durbin R. Fast and accurate long-read alignment with Burrows-Wheeler transform. Bioinformatics. 2010; 26:589-95. [PubMed: 20080505]

53. Edge P, Bafna V, Bansal V. HapCUT2: robust and accurate haplotype assembly for diverse sequencing technologies. Genome Res. 2017; 27:801-812. [PubMed: 27940952] 
54. Dignam JD, Lebovitz RM, Roeder RG. Accurate transcription initiation by RNA polymerase II in a soluble extract from isolated mammalian nuclei. Nucleic Acids Res. 1983; 11:1475-89. [PubMed: 6828386]

55. Cox J, Mann M. MaxQuant enables high peptide identification rates, individualized p.p.b.-range mass accuracies and proteome-wide protein quantification. Nat Biotechnol. 2008; 26:1367-72. [PubMed: 19029910]

56. Khan A, et al. JASPAR 2018: update of the open-access database of transcription factor binding profiles and its web framework. Nucleic Acids Res. 2018; 46:D260-D266. [PubMed: 29140473] 


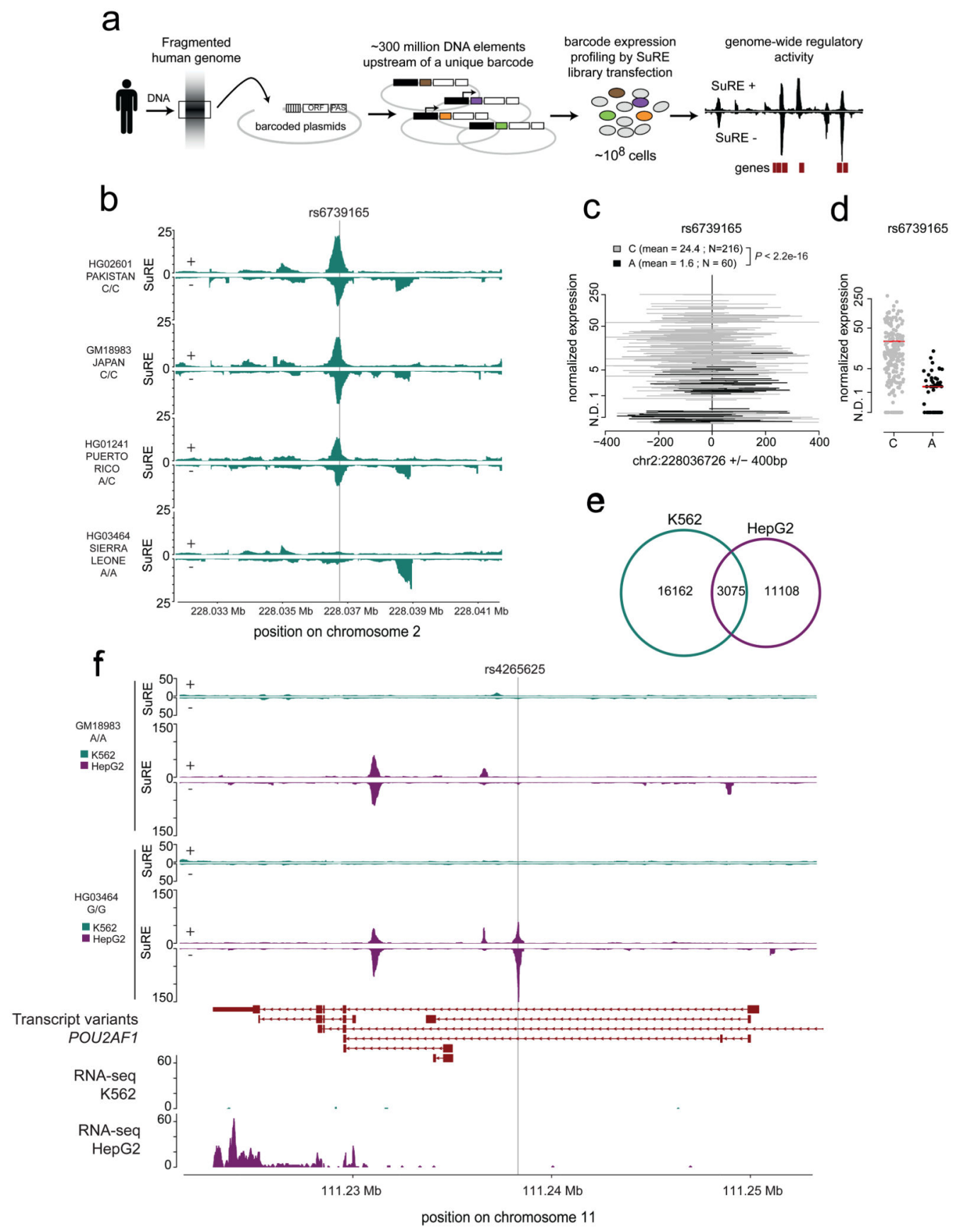

Fig. 1. Identification of raQTLs by SuRE.

a. Schematic representation of the SuRE experimental strategy. ORF, open reading frame; PAS, polyadenylation signal. Colors indicate different barcodes. SuRE yields orientationspecific activity information26 (SuRE +/- tracks, right-hand panel). b. SuRE signals from the four genomes in an example locus, showing differential SuRE activity at raQTL rs6739165, depending on the allele (A or C) present. c. SuRE activity for all fragments containing rs6739165. N.D., not detected. SuRE data of + and - orientations are combined. Values on the $y$-axis were shifted by a random value between -0.5 and 0.5 in order to better 
visualize DNA fragments with the same value. $P<2.2 \times 10^{-16}$, according to two-sided Wilcoxon rank-sum test. d. Same data as in (c), but only the expression value for each fragment is shown (without the addition of the random value). Red lines indicate mean values. e. Numbers of raQTLs in K562, HepG2, or both. f. Example of a locus showing differential SuRE activity for 2 genomes in HepG2 only. Below the SuRE tracks known transcript variants of $P O U 2 A F 1$ are indicated, and RNA-seq data from K562 and HepG2 (data from28). 

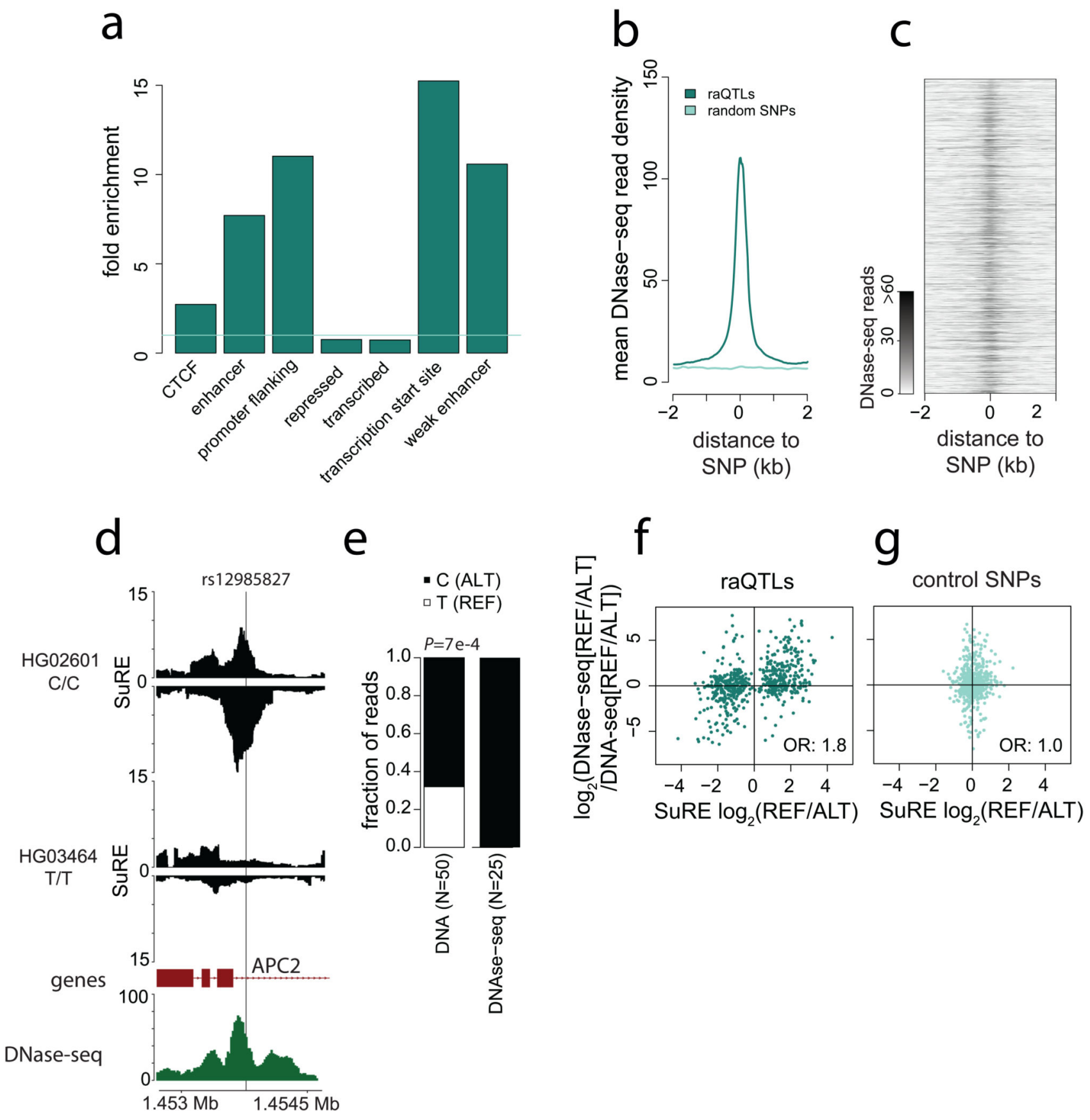

Fig. 2. Correlation of SuRE signals with local chromatin states.

a. Enrichment or depletion of 19,237 raQTLs among major types of chromatin in K56228 relative to all SNPs analyzed. All values are significantly different from $1\left(P<2.2 \times 10^{-16}\right.$, two-sided Fisher exact test). b. Average profile of DNase-seq enrichment for the 19,237 raQTLs compared to an equally sized random set of analyzed SNPs. c. DNase-seq signals aligned to the 19,237 raQTLs, sorted by their $P$ value according to our SuRE analysis (lowest $P$ value on top). d-e. Example of a SNP with differential SuRE activity for the two alleles, overlapping with a DNase-seq peak in K562 cells (d) and showing only DNase 
sensitivity for one allele, even though both alleles are present in the K562 genome. Note that K562 cells are aneuploid, hence the balance of the alleles in input DNA may not be 1:1 (e). $P$ value according to two-sided Fisher exact test. f. Comparison of allelic imbalance of SuRE signals and DNase-seq signals (normalized for genomic DNA allelic read counts) for 616 raQTLs for which K562 cells are heterozygous. REF: reference allele; ALT: alternative allele; OR: odds-ratio. g. Same as in (f) but for a random set of 616 control SNPs overlapping with a DNase-seq peak. DNase-seq data in b-g are from 28 . 

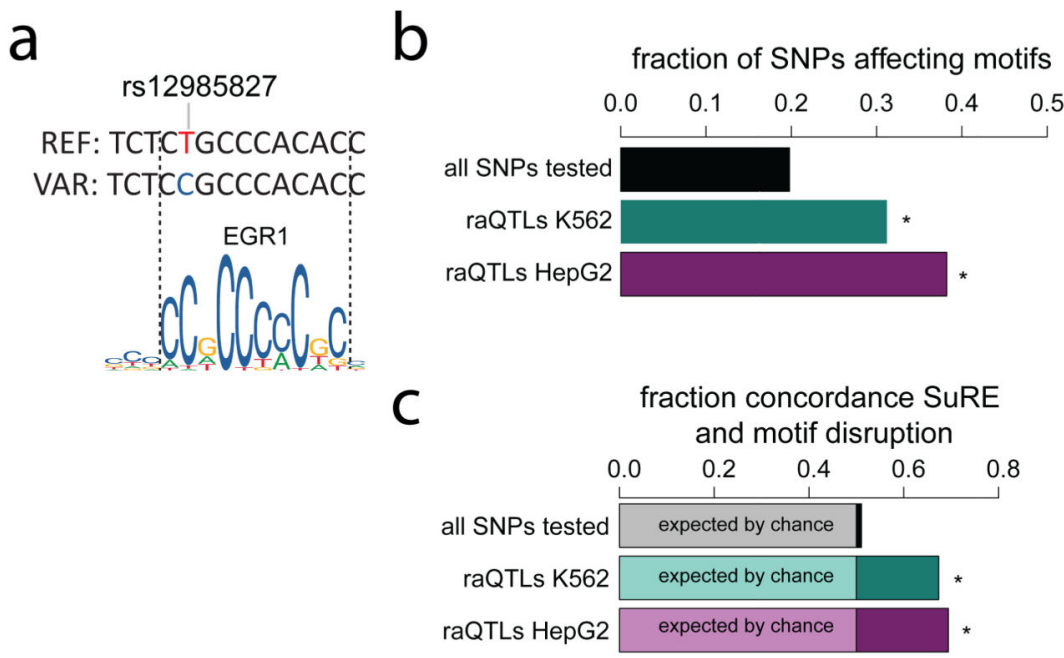

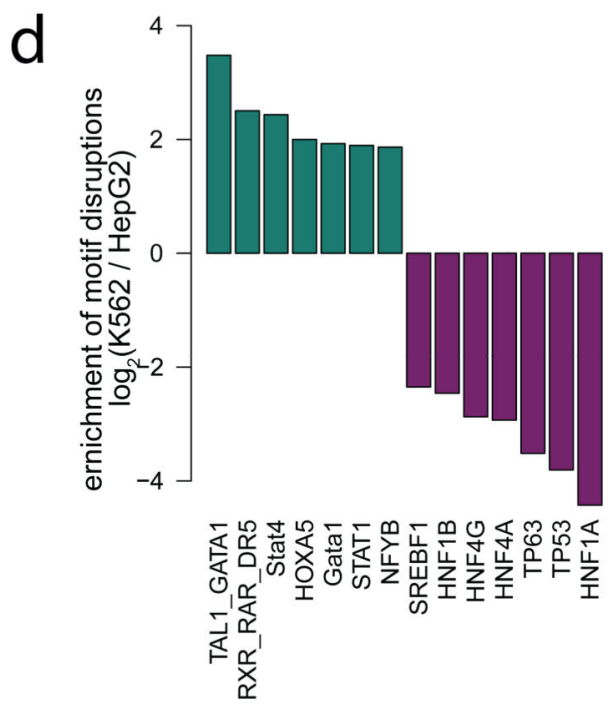

Fig. 3. Concordance of SuRE data and predictions based on TF binding motifs.

a. Comparison of the sequence flanking raQTL rs12985827 (same SNP as in Fig. 2d-e) and the sequence logo for EGR1. The T allele disrupts a conserved nucleotide in the EGR1 binding motif. b. Compared to all SNPs $(n=5,919,293)$, raQTLs in K562 ( $n=19,237)$ and HepG2 $(n=14,183)$ both overlap preferentially with computationally predicted alterations of TF binding motifs according to SNP2TFBS30. Asterisks, $P<2.2 \times 10^{-16}$, according to two-sided Fisher exact test. c. Concordance between the predicted increase or decrease in TF binding according to SNP2TFBS, and the observed effect in SuRE, assuming that decreased TF binding typically leads to decreased activity of a regulatory element. Asterisks, $P<2.2 \times$ $10^{-16}$, according to two-sided Fisher exact test. d. TF motif alterations that are preferentially present among raQTLs in either K562 or HepG2 cells. Only the 7 most enriched TF motifs for each cell type are shown. 
a

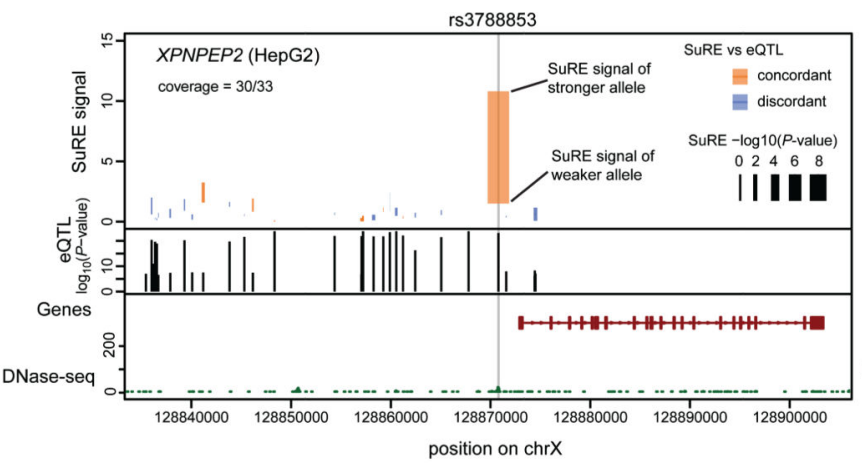

b
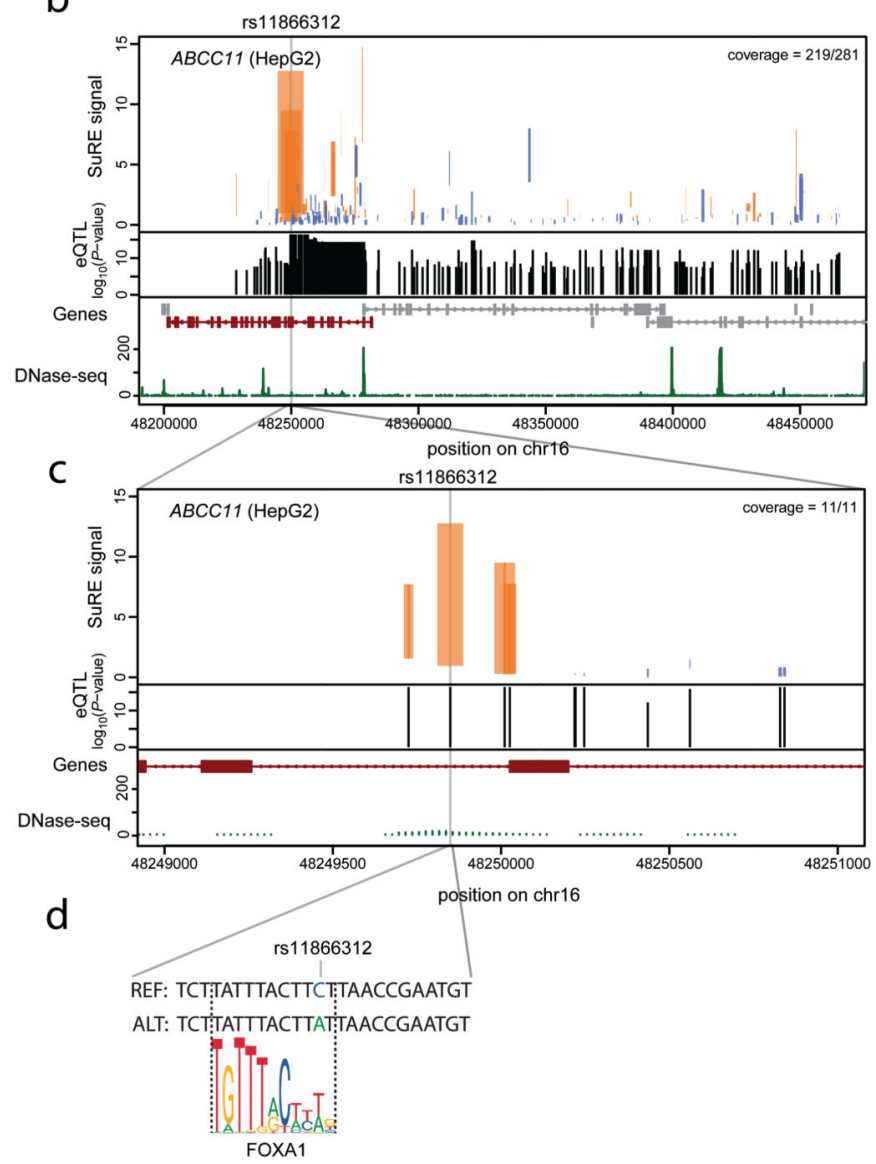

e

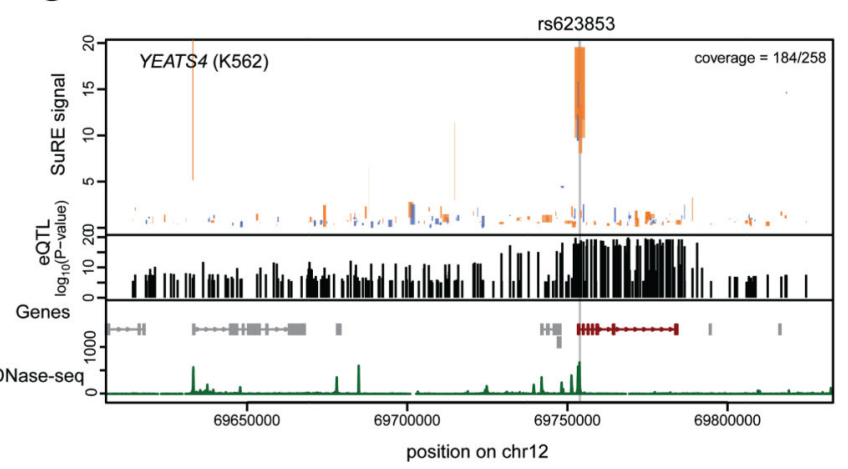

f

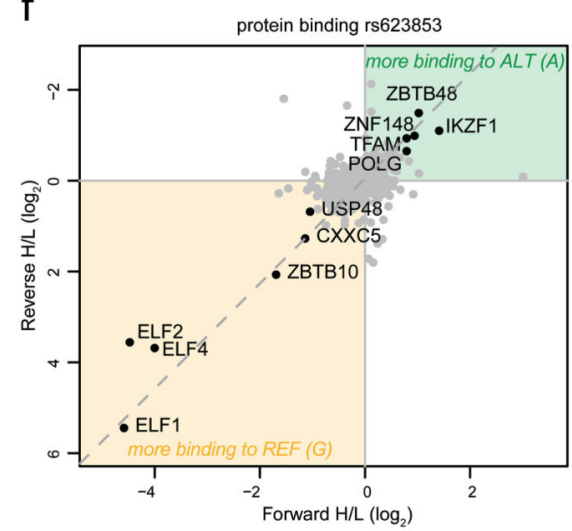

9

rs623853

REF: CGCCCGTCTTCC GGGTGGCTCTCC ALT: CGCCCC:TCTTCCAGGG:GGCTCTCC

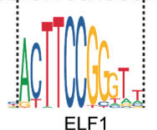

Fig. 4. Candidate causal SNPs identified by SuRE among large sets of eQTL SNPs. a. SuRE signals in HepG2 cells for eQTL SNPs previously identified for the XPNPEP2 gene in liver according to GTEx v78. Top panel: SuRE data in HepG2 cells. Top and bottom of each bar indicate the SuRE signal of the strongest and weakest allele, respectively. Width of the bars is proportional to the $-\log _{10}(P$ value $)$ obtained by a two-sided Wilcoxon rank-sum test; color indicates whether the eQTL effect orientation is concordant or discordant with the SuRE effect orientation. Middle panel: positions of significant eQTL SNPs with the associated eQTL $-\log _{10}(P$ values) according to GTEx v78. Bottom panel: gene annotation of $X P N P E P 2$ and DNase-seq data from HepG2 cells28. b. Same as (a), but for $A B C C 11$ (dark 
red). c. Zoom in of (b). d. Sequence of rs $11866312 \pm 12$ bp aligned to the binding motif of FOXA1. e. Same as (a) but for YEATS4 with SuRE data from K562 cells and eQTL data from whole blood (GTEx v78). In (a-c, e), eGenes are shown in the bottom panels in dark red and all other genes in gray; coverage numbers in the top panels indicate the number of SNPs with SuRE data out of the total number eQTL SNPs. f. Mass-spectrometry analysis of proteins from a K562 cell extract binding to 25-bp double-stranded DNA oligonucleotides containing either the $\mathrm{A}$ or $\mathrm{G}$ allele of rs623853. The experiment was performed once with heavy labeling of proteins bound to the A allele and light labeling of proteins bound to the $G$ allele (x-axis), and once with reverse labeling orientation (y-axis). g. Sequence of the DNA probes used in (f) aligned to the binding motif of ELF156. 


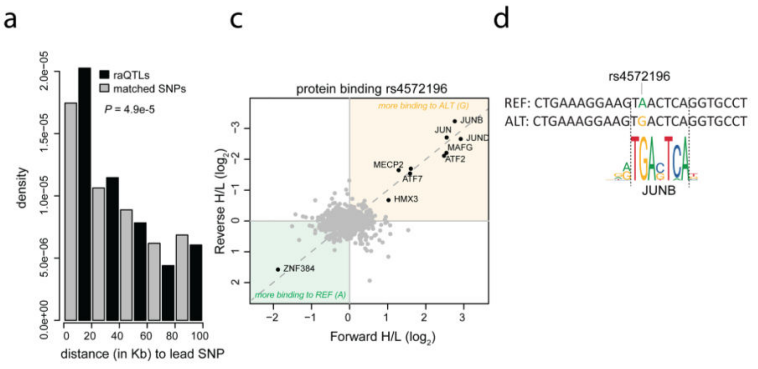

b
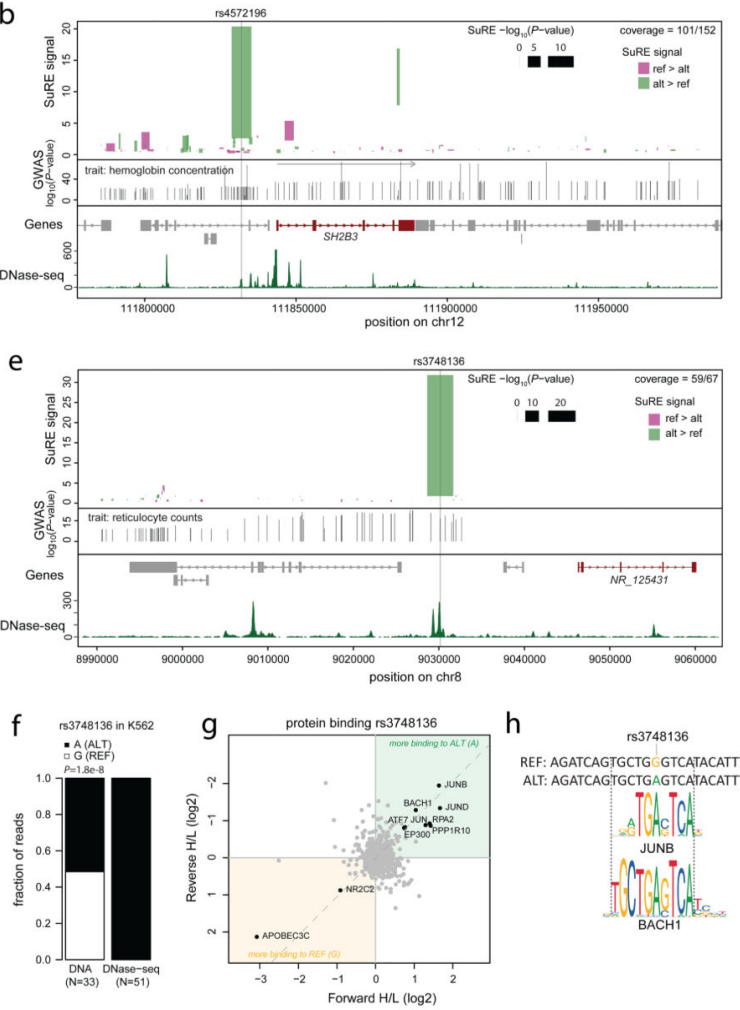
REF: AGATCAGTGCTGGGTCATACATTTC
ALT: AGATCAGTGCTGAGTCATACATTTC

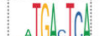

A $A=C \mid \mathrm{C} / \mathrm{X}$

$\operatorname{MUNB}$

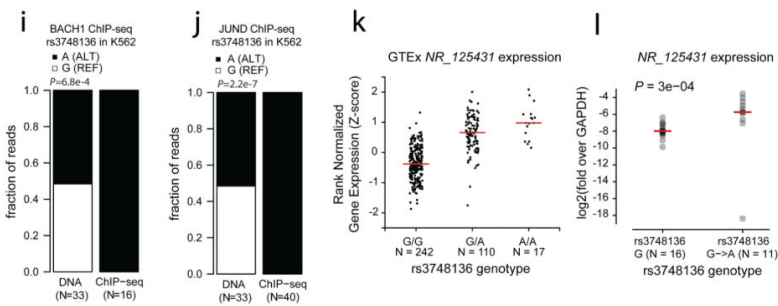

Fig. 5. Candidate causal SNPs identified by SuRE among large sets of GWAS SNPs.

a. Distribution of distances between lead SNPs for blood traits39 and raQTLs (black) and a set of matched control SNPs (gray). $P$ value was obtained with a two-sided Wilcoxon ranksum test. raQTLs in K562 cells are modestly enriched near blood GWAS lead SNPs. $P$ value was obtained using two-sided Wilcoxon rank-sum test. b. Overlay of SuRE and GWAS data for a cluster of GWAS SNPs linked to hemoglobin concentration39. Top panel: SuRE data in K562 cells. Top and bottom end of each bar indicate the SuRE signal of the strongest and weakest allele, respectively. Color of the bars indicates which allele is stronger. Width of the 
bars is proportional to $-\log _{10}(P$ value $)$. Middle panel: positions of significant GWAS SNPs with the associated $-\log _{10}(P$ values $) 39$ on the y-axis. Bottom panel: gene annotation (dark red: $S H 2 B 3$ ) and DNase-seq data from K562 cells28. c. Protein binding analysis as in Fig. 4 f, for rs4572196. d. Sequence of the probes used in (c) aligned to sequence logo for JUNB. e. Same as (b) but for a cluster of SNPs associated with reticulocyte counts by GWAS39. f. Fraction of reads containing each of the two alleles of rs3748136 in K562 genomic DNA and K562 DNase-seq reads. $P$ value was obtained with a two-sided Fisher exact test. g. Same as (c) but for rs3748136. h. Sequence of the probes used in (g) aligned to binding motifs for JUNB and BACH1. i. Fraction of reads containing each of the two alleles of rs3748136 in K562 genomic DNA (left) and K562 ChIP-seq reads for BACH1 (right). j. Same as (i) but for ChIP-seq reads for JUND. ChIP data are from28. k. Association between alleles of rs3748136 and $N R_{-} 125431$ expression in whole blood according to GTEx8. Red lines indicate median. I. Expression of $N R \_125431$ in subclones derived from K562 clone BL_2 subjected to CRISPR/Cas9 editing of rs 1053036. Sixteen unaltered subclones and eleven G$>$ A edited subclones were assayed by RT-qPCR of $N R \_125431$ (normalized to GAPDH). $P$ value was obtained with a two-sided Wilcoxon rank-sum test. Red lines indicate medians. One G->A subclone appeared to have reverted to the completely inactive state seen in many K562 clones initially derived from the cell pool (Supplementary Fig. 5c). 


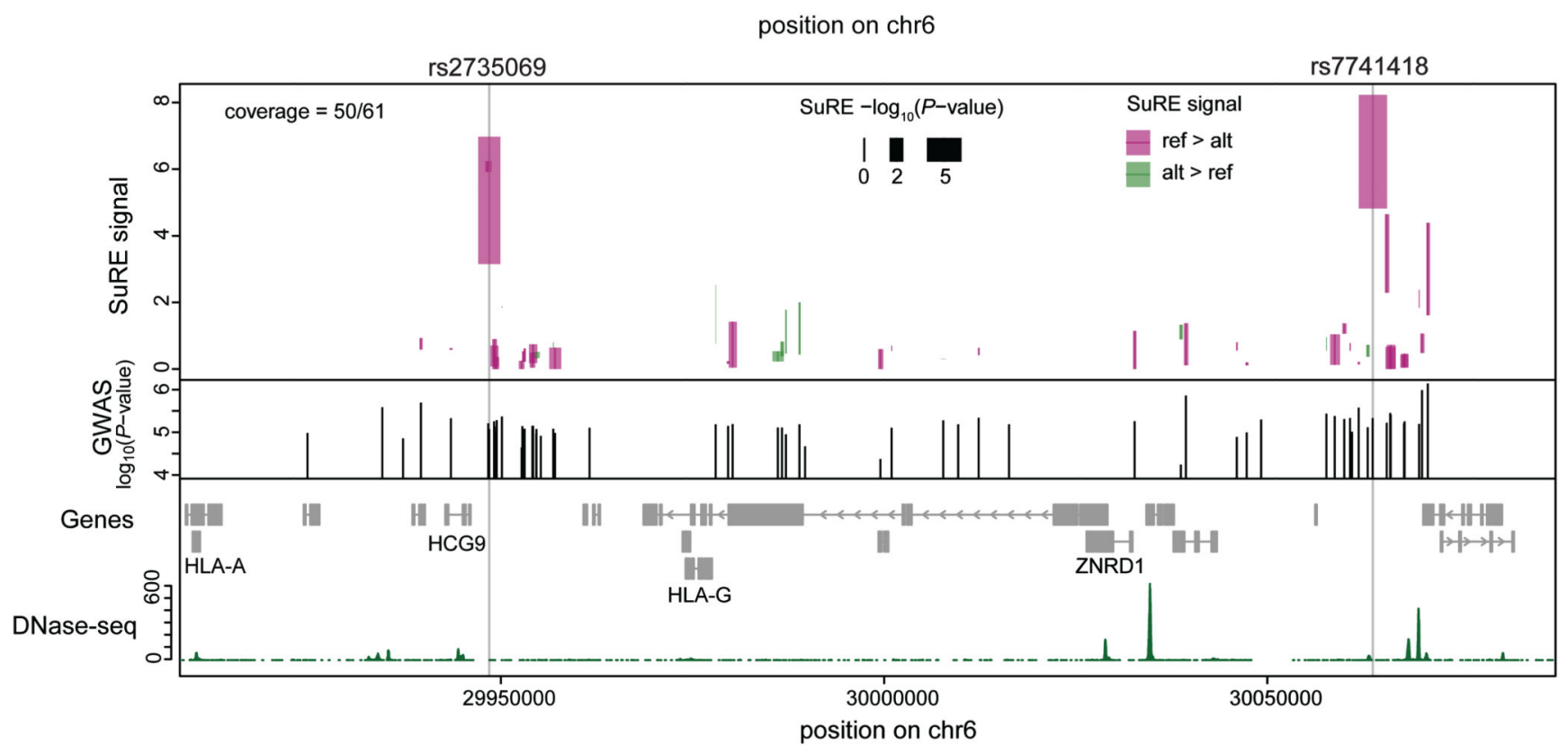

Fig. 6. Candidate causal SNPs identified by SuRE among GWAS SNPs for hepatocellular carcinoma.

Comparison of SuRE and GWAS data for a cluster of GWAS SNPs linked to hepatocellular carcinoma42. Top panel: SuRE data in HepG2 cells. The top and bottom end of each bar indicate the SuRE signal of the strongest and weakest allele, respectively. Color of the bars indicates which allele is stronger. Width of the bars is proportional to $-\log _{10}(P$ value $)$ obtained by a two-sided Wilcoxon rank-sum test. Middle panel: positions of significant GWAS SNPs with the associated $-\log _{10}(P$ values $) 42$ on the y-axis. Bottom panel: gene annotation track and DNase-seq data from HepG2 cells28. 